\title{
Anabases
}

ANABASES Traditions et réceptions de l'Antiquité

$24 \mid 2016$

Varia

\section{Sarcophages et klinai. À propos de la représentation du couple dans l'art grec}

\section{Alain Pasquier}

\section{(2) OpenEdition}

\section{Journals}

Édition électronique

URL : http://journals.openedition.org/anabases/5916

DOI : 10.4000/anabases.5916

ISSN : 2256-9421

\section{Éditeur}

E.R.A.S.M.E.

\section{Édition imprimée}

Date de publication : 10 novembre 2016

Pagination : 107-124

ISSN : 1774-4296

\section{Référence électronique}

Alain Pasquier, "Sarcophages et klinai. À propos de la représentation du couple dans l'art grec », Anabases [En ligne], 24 | 2016, mis en ligne le 01 octobre 2019, consulté le 22 mars 2020. URL : http:// journals.openedition.org/anabases/5916 ; DOI : https://doi.org/10.4000/anabases.5916 


\section{Sarcophages et klinai. À propos de la représentation du couple dans l'art grec}

Alain PAsQuier

I y a bien longtemps que l'on a remarqué, et amplement commenté, l'influence des modèles grecs, particulièrement ioniens, dans les formes des époux à demi couchés sur la klinè des grands sarcophages de Cerveteri, au Louvre (fig. 1) et au Musée de la Villa Giulia. Mais dans cet art grec auquel il est banal de rappeler que les Etrusques doivent beaucoup, on pourrait ingénument se demander s'il existe des sarcophages semblables. Bien sûr, personne, surtout ici, n'ignore que tel n'est pas le cas. L'inspiration de l'artiste cérétain qui a modelé les visages des deux couples était certes tout imprégnée des motifs que l'on découvre sur les vases peints de la côte occidentale micrasiatique, de Phocée, de Clazomènes ou de leur région. Mais disons-le tout net : le projet de tels sarcophages n'a pas d'équivalent grec.

Cela ne veut pas dire qu'il n'existe pas de sarcophage grec. Dans un ensemble de coutumes funéraires d'une foisonnante diversité, où l'inhumation cohabite souvent avec la crémation, le sarcophage est un mode d'ensevelissement qui demeure luxueux, surtout, bien sûr, s'il est décoré : mais s'il l'est, ce n'est jamais selon cette mode des Etrusques dont les deux monuments de Caeré nous donnent à voir de majestueux exemples. Parlons-en rapidement. Les sarcophages grecs, qu'on rencontre dès la période mycénienne (fig. 2$)^{1}$, se répartissent en une grande variété de formules, selon les époques et les lieux. Ils peuvent être en terre cuite, comme ceux de Caeré, mais aussi en bois et en

1 Sur les sarcophages mycéniens, voir J.-C. Poursat, L'Art égéen 2. Mycènes et le monde mycénien, Paris 2014, p. 221-239. Pour les sarcophages grecs, en général : J. BoardmanD.C. Kuntz, Greek Burial Customs, Londres 1971, en particulier p. 267-272, et 308-310. 


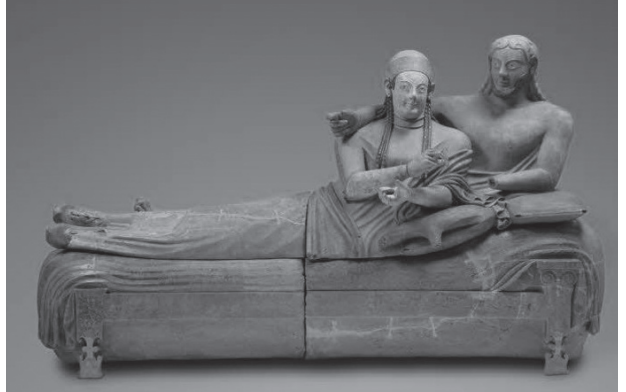

Fig. 1. Le sarcophage des époux, Musée du Louvre.

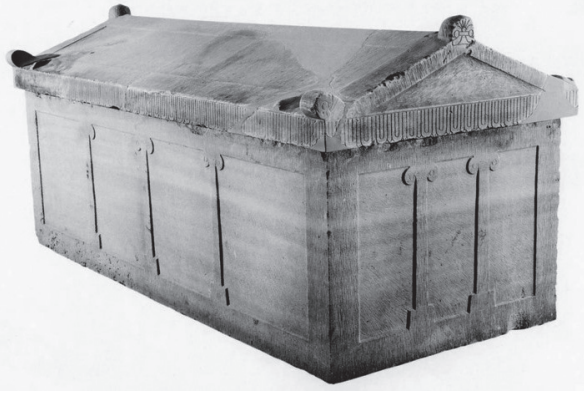

Fig. 3. Sarcophage en marbre, Samos, vers 550 av. J.-C.

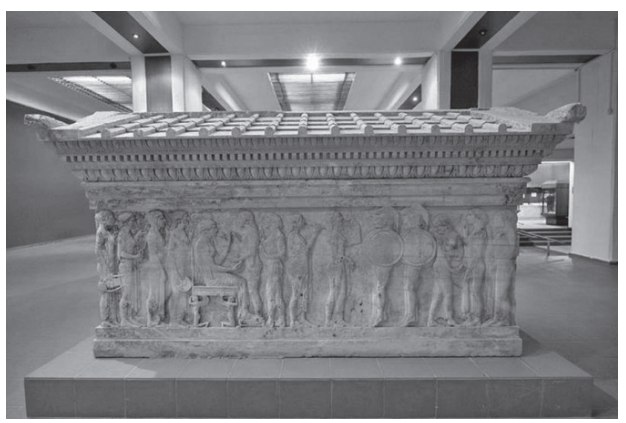

Fig. 5. Sarcophage en marbre, Musée de Çannakale, fin du VIe siècle av. J.-C.

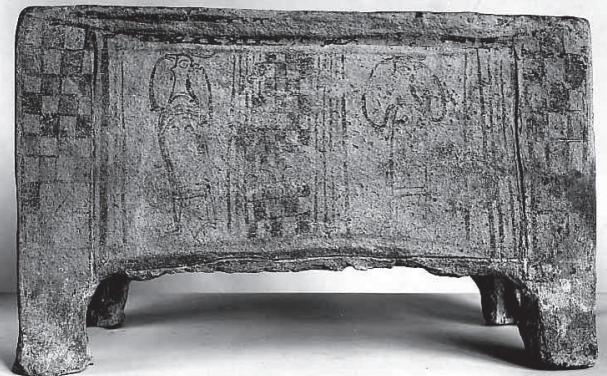

Fig 2. Sarcophage (larnax) mycénien en terre cuite à décor de pleureuses, Tanagra, Louvre CA 6988, vers 1300-1250 av. J.-C.

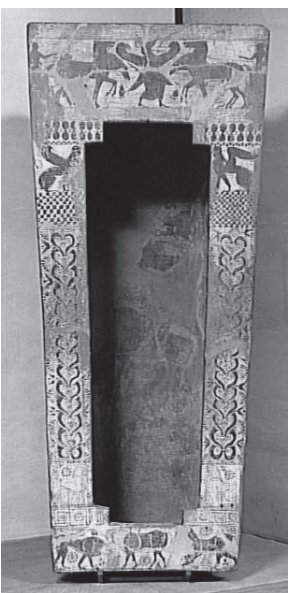

Fig. 4. Sarcophage de Clazomènes en terre cuite, Louvre CA 460 bis, vers 510 av. J.-C.

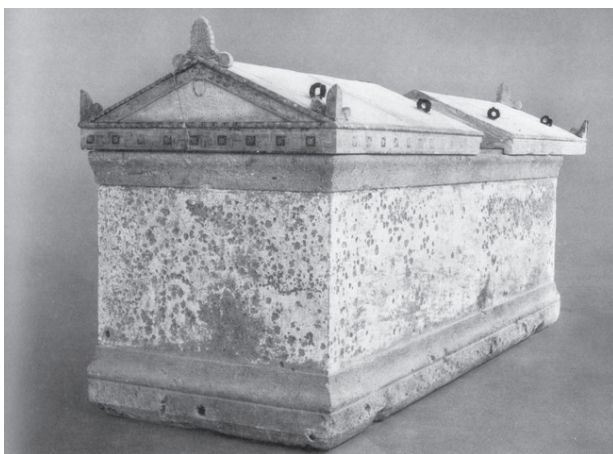

Fig. 6. Sarcophage en marbre peint, Musée national de Tarente, début du Ve siècle av. J.-C. 
pierre. Pour rester dans la proximité chronologique des objets qui nous ont rassemblés à Amiens, citons l'exemple d'un sarcophage trouvé à Samos², en pierre celui-là, qui renvoie l'image d'un temple, avec des colonnes ioniques taillées en bas-relief aux angles du coffre (fig. 3) : il date du milieu du VI ${ }^{e}$ siècle av. J.-C., mais n'a aucun rapport avec les cercueils étrusques. On a remarqué que cette mode funéraire était plus répandue en Grèce de l'est. Nombreux sont en effet ceux qui, en terre cuite et soigneusement ornés, sont dénommés « sarcophages de Clazomènes ", quoiqu'on puisse les trouver aussi à Ephèse, à Smyrne, à Lesbos ou Chios, voire à Rhodes (fig. 4) ${ }^{3}$. Cependant le décor y est uniquement peint, à la manière des vases de la même période. Si l'on veut surtout retenir les effets plastiques, il faut alors mentionner l'étonnant sarcophage en marbre de Proconnèse trouvé dans la vallée du Granique et conservé au musée de Çanakkale $(\text { fig. 5 })^{4}$. Ses reliefs peuplés d'une foule de personnages, parmi lesquels se rencontre la fascinante et cruelle image du meurtre de Polyxène, posent de passionnantes questions, tant du point de vue du sens que du style, ou des styles. Mais si la date à laquelle il est plus que probable qu'il a été créé coïncide avec l'apparition des grands sarcophages de Caeré, il n'a rien de commun avec eux, si ce n'est la fonction funéraire. La même remarque vaut pour un sarcophage en marbre peint de Tarente (fig. 6 et 7) ${ }^{5}$, un peu plus tardif, qui ne fait que reproduire l'architecture d'un temple, avec frontons et acrotères. Et sans quitter Tarente, mais en glissant au sein du IVe siècle, on rencontre des éléments de décor pour sarcophages en bois, sous la forme d'appliques en terre cuite dorée qui étaient fixées sur les parois du coffre (fig. 9) ${ }^{6}$. En bois aussi, des sarcophages exhumés des terres coloniales de la Russie méridionale, ornés de plaquettes dorées et de statuettes (fig. 8) ${ }^{7}$. Aucune équivalence avec les objets de notre colloque.

2 EAA V, $2^{e}$ supplement, 1971-1994, s.v. sarcofago, p. 109-114, fig. 137 (G. Косн). K. Tsakos - M. Viglaki-Sofianou, Samos - The Archaeological Museums, John S. Latsis, Public Benefit Foundation, Athènes 2012, p. 214-215.

3 R.M. Cоок, "Clazomenai Sarcophagi", Kerameus - Forschungen zur antiken Keramik 2. S. III, Magonza 1981.

4 Ch.B. Rose, The Archeology of Greek and Roman Troy, Cambridge 2013, ch. 3. Cf. Fr. Croissant, Revue Archéologique 2015.2, p. 259-292.

5 Megale Hellas - Storia e civiltà della Magna Grecia, Milan 1983 (Credito Italiano), p. 711713, fig. 711 et 717 (L.ForTi - A.Stazio); G.P. Carratelli, éd., Magna Grecia-Lo sviluppo politico, sociale ed economico, Milan 1987, p. 43, fig. 48 et 49 (M. Giangiulio).

6 R. Lullies, "Vergoldete Terrakotta-Appliken aus Tarent », Römische Mitteilungen, Suppl. XVII. Cf. Catalogo del Museo Nazionale Archeologico di Taranto, III, 1, Tarente 1994, p. 130-147 (E. LipPolis).

7 P. Pinelli -A. Wasowicz, Catalogue des bois et stucs grecs et romains provenant de Kertch. Musée du Louvre. Paris 1986, p. 31-48. C. Watzinger, Griechische Holzsarcophage, Leipzig 1905. 


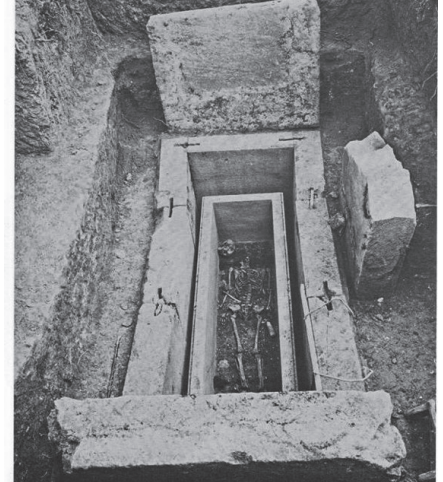

Fig. 7. Le sarcophage de Tarente in situ.

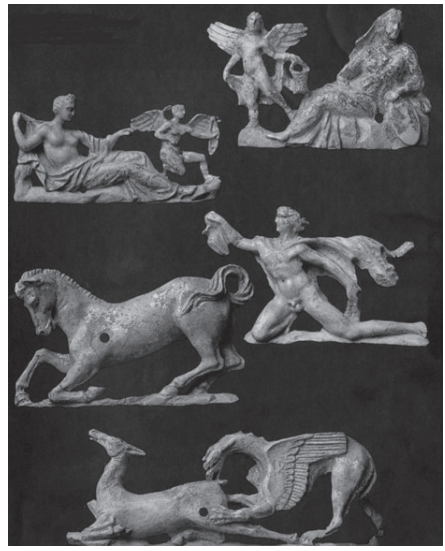

Fig. 9. Appliques en argile dorée du décor d'un sarcophage, Musée National de Tarente, $\mathrm{IV}^{\mathrm{e}}$ siècle av. J.-C.

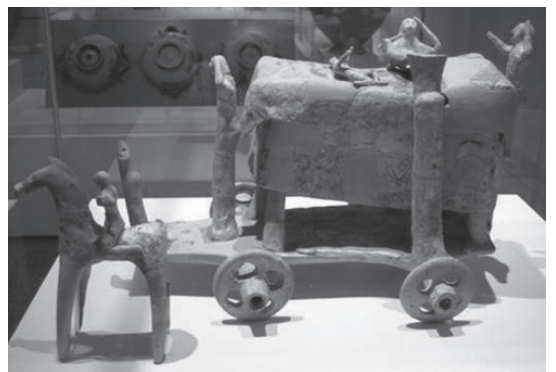

Fig. 11 Char funéraire transportant le lit mortuaire (klinè), Anagyrous (Vari), Musée National d'Athènes, vers 650-630 av. J.-C.

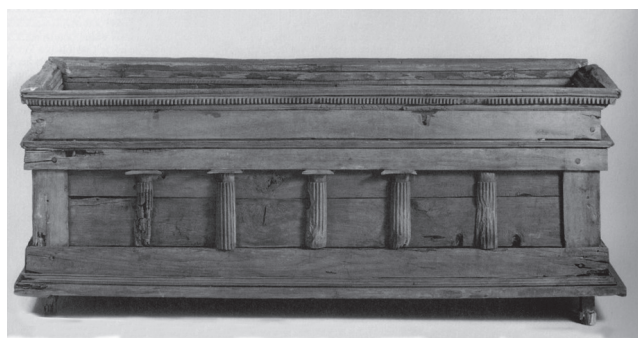

Fig. 8. Sarcophage en bois d'un enfant, de Kertch, Musée de Berlin, Misc 10477, $\mathrm{IV}^{\mathrm{e}}$ siècle av. J.-C.

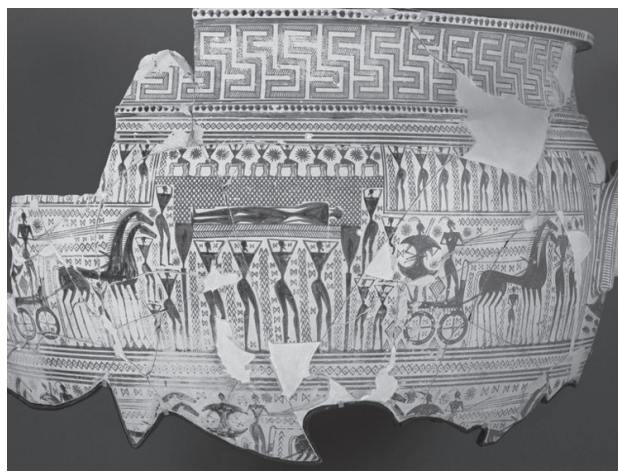

Fig. 10. Cratère géométrique attique, Louvre A 517, vers 760-750 av. J.-C.

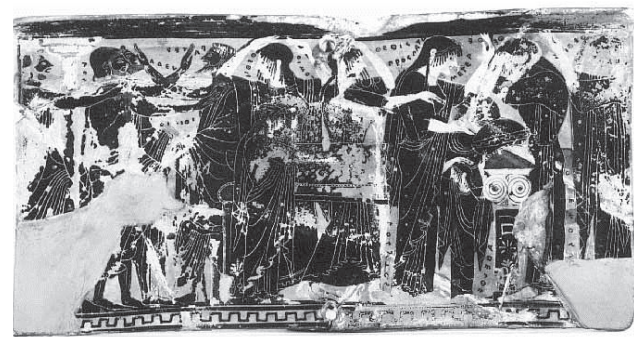

Fig. 12. Plaque funéraire attique à figures noires : décor attribué au Peintre de Sappho, Louvre MNB 905, vers 500 av. J.-C. 
Ceux-ci réunissent, comme on le sait, la forme du sarcophage contenant les restes des époux, et la klinè sur laquelle ceux-ci sont figurés dans l'attitude des banqueteurs. Là encore, les klinai funéraires ne manquent pas chez les Grecs, auxquels les Etrusques doivent l'usage rituel du banquet, que les Grecs eux-mêmes avaient emprunté à l'Orient. Les vases de l'époque géométrique ne manquent pas de ces scènes de prothésis où le mort est allongé sur une klinè (fig. 10) ${ }^{8}$, laquelle peut être transportée dans le rite de l'ekphora, comme le montre le groupe en terre cuite de Vari (fig. 11) ${ }^{9}$. Mais pour maintenir une proximité chronologique avec les tombeaux de la nécropole étrusque, mentionnons la présence d'une élégante klinè dans une sépulture enfouie sous un tumulus du cimetière du Céramique : il s'agirait probablement de la tombe d'un ambassadeur envoyé à Athènes ${ }^{10}$; citons aussi, entre autres décors du même genre, un pinax attique à figures noires attribué au Peintre de Sapho qui montre le mort allongé sur son lit funèbre, entouré de toute sa famille (fig. 12). Cependant c'est évidemment dans les tombes dites “ macédoniennes », beaucoup plus tardives, que les klinai funéraires abondent. On ne les rencontre pas seulement en Macédoine, comme le montre par exemple la tombe d'Amarynthos, en Eubée (fig. 13) ${ }^{11}$. Toutefois si ces klinai pouvaient soit supporter le corps du défunt, soit même contenir ses restes dans un compartiment qui y était aménagé, l'effigie du disparu n'était pas pour autant sculptée sur leur couvercle. D'ailleurs, en dehors de l'art étrusque, qui a continué à pratiquer cette mode funéraire, il semble qu'il faille attendre l'époque de Trajan pour retrouver des sarcophages supportant la statue-portrait du mort ou du couple des défunts représentés à demi couchés sur leur klinè (fig. 15) ${ }^{12}$.

Avant de cesser cette errance un peu vaine dans le domaine sans limites des usages que les Grecs ont empruntés pour honorer la dépouille de leurs morts, observons qu'il n'y a en définitive qu'un monument qui puisse à la rigueur passer pour un écho grec aux sarcophages étrusques, un écho lointain, affaibli,

8 Voir, par exemple, M. Denoyelle, Chefs-d'œeuvre de la céramique grecque, Musée du Louvre, Paris 1994, p. 18-19.

9 P. Demargne, Naissance de l’Art grec, Paris 1974, réédité en 2007, p. 292, fig. 198. A. Alexandridou, Special burial treatment for "heroized " deceased at the Attic countryside (en ligne).

10 E.P. Baugnan, Couched in Death - Klinai and Identity in Anatolia and Beyond, University of Wisconsin Press 2013, p. 62-63, fig 43-44, et p. 225. Cf. U. Knigge, "Der Südhügel”, Kerameikos BdIX, Berlin 1976.

1 C. Huguenot, « La tombe aux Erotes et la tombe d'Amarynthos », Eretria XIX, Université du Michigan 2008.

12 H. Wrede, "Der Sarkophagdeckel eines Mädchens in Malibu und die frühen Klinensarkophage Roms, Athens und Kleinasiens", Roman Funerary Monuments in the J.P. Getty Museum, volume I, Malibu 1990, p. 15-46. 


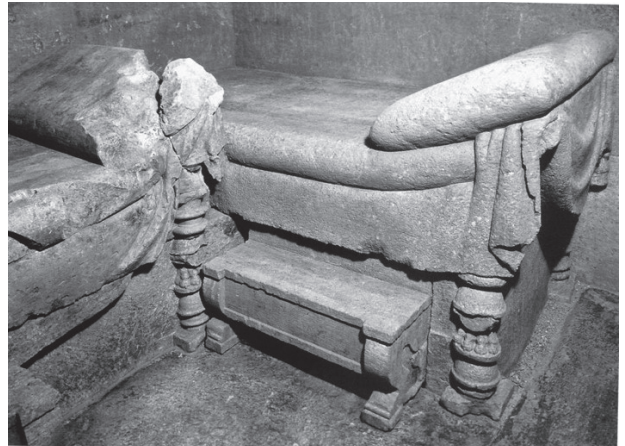

Fig. 13. Couches funéraires (klinai) à l'intérieur de la tombe d'Amarynthos, Eubée (Grèce), deuxième moitié du III ${ }^{\mathrm{e}}$ siècle av. J.-C.

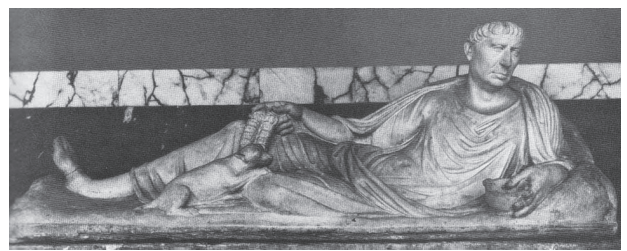

Fig. 15. Sarcophage romain avec le défunt sculpté sur la klinè. Copenhague, Ny Carlsberg Glyptotek Sk. 777, vers 130.

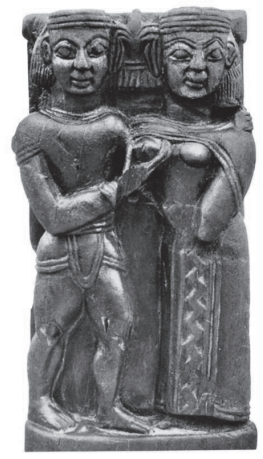

Fig. 17. Relief en bois, autrefois à Samos, vers 630-600 av. J.-C.

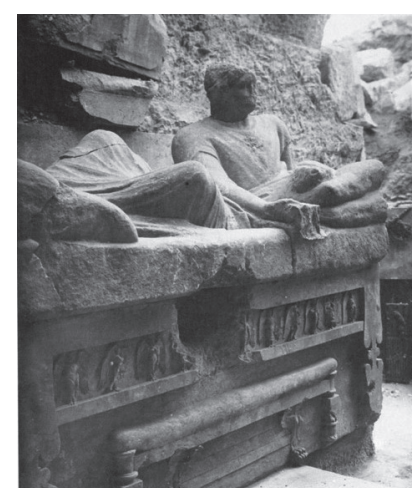

Fig. 14. Sarcophage en forme de klinè sur laquelle repose le défunt, Musée de Selçük, vers 246 av. J.-C.

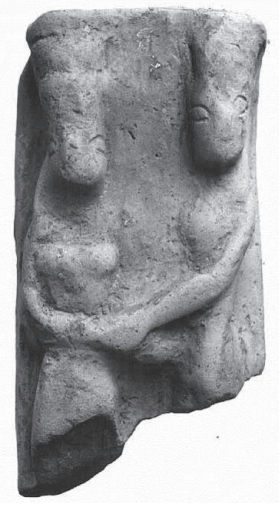

Fig. 16. Relief en terre cuite, Praesos, Louvre AM 848, vers 645 av. J.-C.

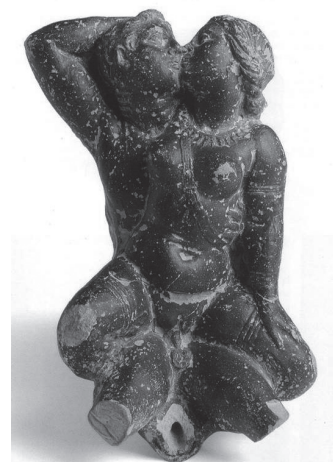

Fig. 18 Décor d'un vase en terre cuite, Musée de Délos B 7461, II ${ }^{e}$ siècle av. J.-C. 
et probablement fortuit : il s'agit du sarcophage en marbre que beaucoup croient pouvoir identifier comme celui du souverain séleucide Antiochos II Théos, et que contenait dans une chambre basse la tombe monumentale de Belevi, proche d'Ephèse, datant du milieu du III ${ }^{\mathrm{e}}$ siècle av. J.-C. (fig. 14) ${ }^{13}$ Prévue d'abord pour Lysimaque, celle-ci échut à Antiochos II, alors qu'elle n'était pas achevée (elle ne le sera jamais), par les soins de sa femme Laodice. Mais cette dernière ne figure pas sur le couvercle où son royal époux est représenté couché sur une klinè, dans l'attitude du banqueteur.

Laissons donc ce monument inachevé, où la tête endommagée est très incomplète. Abandonnons même les tombeaux grecs où il est assurément vain de retrouver le dispositif brillamment illustré par nos deux couples de Cerveteri, et essayons-nous à méditer quelques instants sur la représentation du couple homme-femme dans le domaine de la plastique grecque. Une représentation dont le répertoire offre, au cours des siècles, un large éventail d'images, depuis celle d'un relief crétois du VII siècle av. J.-C. (fig. 16) jusqu’à un symplegma délien du II ${ }^{\mathrm{e}}$ siècle av. J.-C., où, d'un objet à l'autre, le rapport érotique s'exprime par des moyens bien différents, d'une sorte de pictogramme schématique à l'abandon le plus descriptif et le plus sensuel (fig. 18) ${ }^{14}$. Sur la représentation des couples les plus anciens, empreints des influences de l'Orient, on relève la ferme enjambée de l'homme et sa main conquérante posée avec autorité sur la poitrine de sa compagne. Et à la différence des époux de Caeré dont il est assuré que ce sont des mortels, il ne fait guère de doute que le groupe en bois de Samos met en scène des personnages divins, et que nous avons affaire ici à une scène de théogamie (fig. 17) ${ }^{15}$ : on s'accorde à penser que l'aigle qui s'envole entre les deux têtes désigne Zeus, dans l'acte du mariage sacré qui l'unit à Héra. Transportons-nous maintenant à Sparte, où ont été sculptés les reliefs ornant une base pyramidante (fig. 19) : tandis qu'un serpent lové ondule sur les petits côtés, chacune des surfaces les plus larges du bloc est décorée du motif d'un couple ${ }^{16}$. A deux reprises, l'homme enlace la femme : mais si c'est manifestement le désir qui l'y pousse ici, là, c'est pour mieux assurer le coup que son épée va lui porter. L'association à Sparte de ces deux scènes, une scène de cour amoureuse suivie d'une tentative de

13 Br. Sismondo-Ridgway, Hellenistic Sculpture I, Bristol 1990, p. 187-196.

14 Plaquette crétoise: S. Mollard-Besques, Catalogue raisonné des figurines et reliefs en terre-cuite grecs, étrusques et romains, Musée du Louvre, I, Paris 1954, B 168, p. 30 et pl. XXI. Symplegma de Délos : Ph. Zapheiropoulou, Delos - The Testimony of Museum Exhibits, Halandri 1998, p. 191 et p. 284 n¹97.

15 J. Boardman, La Sculpture grecque archä̈que, Paris 1994 (1 ère éd. Londres 1978), p. 16 et p. 46 , fig. 49.

16 Ibid., p. 76 et p. 116, fig. 124. Cf. N. Kaltsas, éd., Athens - Sparta, Catalogue de l'exposition de New York, Onassis Cultural Center, décembre 2006 - mai 2007, p. 89-90, fig. 1a-1b. 

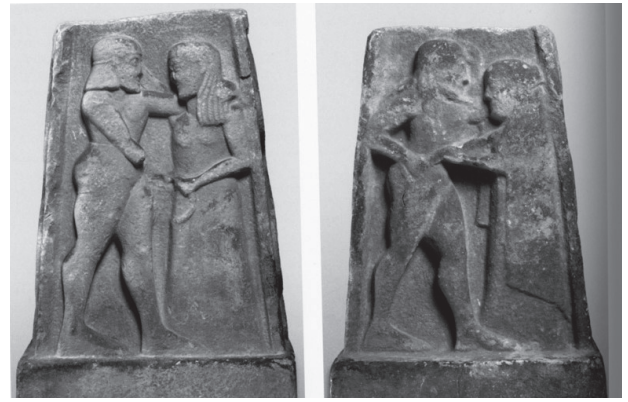

Fig. 19. Monument pyramidal en pierre, Musée de Sparte 1, vers 575-550 av. J.-C.

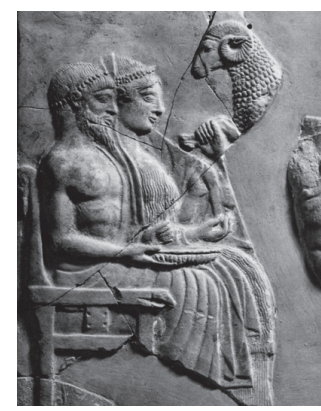

Fig. 21. Pinax en terre cuite,

Musée de Reggio Calabria, vers 460 av. J.-C.

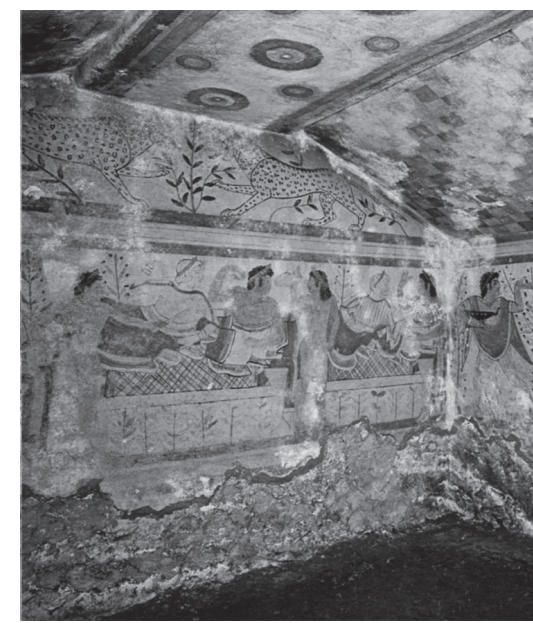

Fig. 23. Fresque de la “ Tombe des Léopards », Tarquinia, vers 450 av. J.-C.

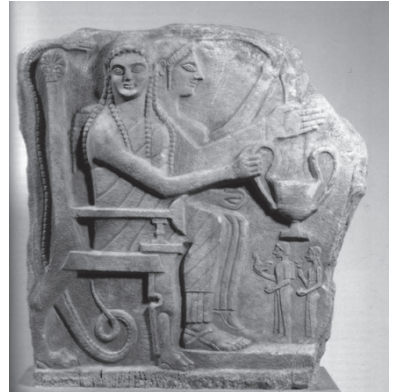

Fig. 20. Relief de Chrysapha (près de Sparte), Musée de Berlin inv. $\mathrm{n}^{\circ} 731$.

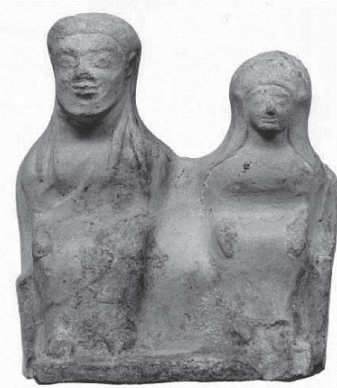

Fig. 22. Groupe en terre cuite de Samos trouvé à Tanagra, Louvre MNB 542, vers 530-520 av. J.-C.

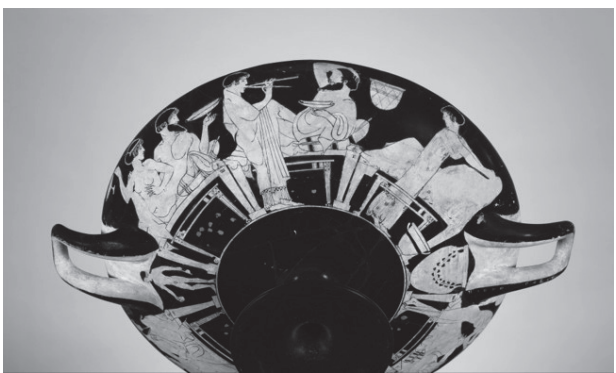

Fig. 24. Coupe attique à figures rouges : scènes de banquet, Metropolitan Museum New York inv. 20.246 (décor attribué à Makron), vers 480 av. J.-C. 
meurtre, devrait désigner Ménélas et Hélène, époux héroïques, mais aussi époux tragiques. Désir impérieux ou désespoir furieux : on est au plus loin de la sérénité harmonieuse des époux Campana.

Des dieux et des héros, donc. Mais il est des représentations du couple hommefemme, dans l'imagerie grecque, dont l'interprétation est plus confuse. Ainsi, arrêtons-nous quelques instants devant le relief du musée de Berlin, trouvé à Chrysapha, près de Sparte, qu'on date généralement du $3^{\mathrm{e}}$ quart du VI ${ }^{\mathrm{e}}$ siècle av. J.-C. (fig. 20) 17. Zeus et Héra, à Samos, Ménélas et Hélène, à Sparte, étaient debout. Voici un homme et une femme assis sur un trône égyptisant derrière lequel un gros serpent se dresse, menaçant. L'homme qui tient un énorme canthare nous regarde, tandis que, de profil, la femme accomplit le geste du dévoilement, l'anakalypsis. Despetits personnagesles approchent, lesmains chargées d'offrandes diverses. On continue à hésiter sur l'identité des figures trônantes : s'agit-il des divinités d'outre-tombe, Hadès et Perséphone ? De figures de la mythologie locale? Ou bien plutôt de mortels héroïsés auxquels on rend hommage ? Le lieu de trouvaille du relief pourrait peut-être valider la troisième hypothèse. Une génération plus tard, une longue série de reliefs en terre cuite trouvés dans le sanctuaire de Perséphone sur le territoire de Locres Epizéphyrienne donne parfois à voir la réunion d'un beau couple trônant côte à côte et auquel on apporte des offrandes (fig. 21 $)^{18}$ : on a voulu y reconnaître Hadès et Perséphone, mais beaucoup penchent plutôt pour Aphrodite et Hermès. L'abondance et la variété des scènes présentées sur les reliefs de la même provenance, où certains rites nuptiaux sont évoqués, en rendent l'interprétation malaisée.

Plus modestes, certaines figurines d'argile, qui brodent à la mode samienne sur le thème du couple, méritent cependant qu'on les prenne en considération (fig. 22). Trouvés à Samos même, mais aussi à Tanagra, à Délos, à Rhodes, et jusqu’à Géla, en Sicile, et Tocra, en Cyrénaïque, ces groupes présentent assis côte à côte, en majesté, un homme barbu et une femme dont la nuque est couverte d'un voile ${ }^{19}$. Ils peuvent susciter les mêmes questions : s'agit-il d'un couple divin, ou bien d'une offrande réunissant soit dans un geste votif, soit dans l'accomplissement d'un rite funéraire, les images réunies de deux mortels ? Leurs attitudes sont d'une dignité empesée, laquelle ne surprend pas sur ces figurines archaïques ; mais la

17 J. Charbonneaux - R. Martin - F. Villard, La Grèce archaïque, Paris 1968, p. 148 et 149, fig. 183.

18 Megale Hellas, op.cit. (ici note 5), p. 449 fig. 470, p. 461-464.

19 Groupe “Zeus-Héra » du Louvre : cf. s. mollard - BesQues, op.cit. (ici note 14), p. 16, B 90, et pl. XI. Voir M. Landolfi, “ La stipe votiva del santuario di Zeus Megistos », dans Missione Archeologica Italiana di Iasos, Iasos e il suo territorio, p. 59-66 et pl. XXXIV (exemples cités de Théangela, Samos, Rhodes, Délos, Tanagra, Géla, Catane, Camarina, Tocra). 


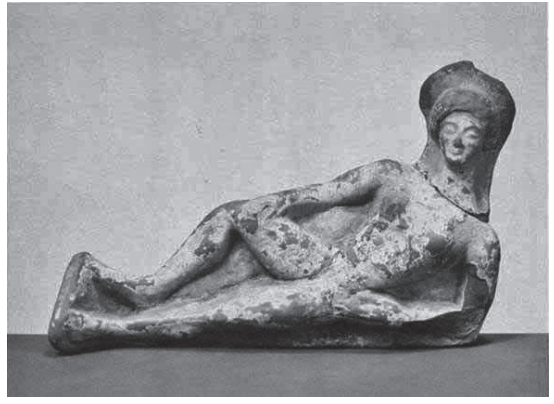

Fig. 25. Statuette attique en terre cuite d'une courtisane au banquet,

Musée de Berlin, Antiquarium inv. 8256, vers 500 av. J.-C.

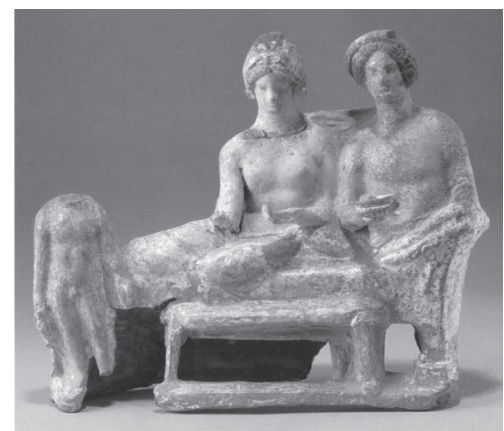

Fig. 27. Groupe attique (?) en terre cuite, Staatliche Antikensammlungen de Munich, Musée de Samos inv. 768, vers 560 av. J.-C. NI 721, vers 400-360 av. J.-C.

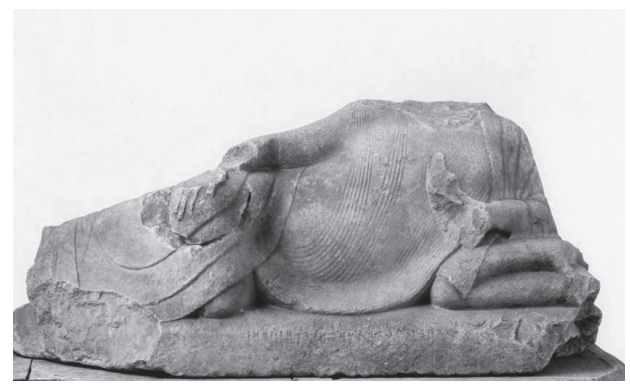

Fig. 29. Groupe de Généléos, [....]ilarchès, Musée de Samos inv. 768, vers 560 av. J.-C.

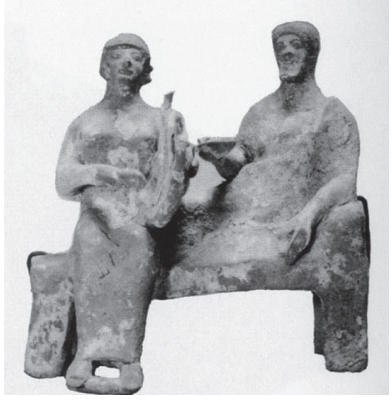

Fig. 26. Groupe béotien en terre cuite, Musée de Dresde inv. ZV 720 a, début du $\mathrm{V}^{\mathrm{e}}$ siècle av. J.-C.

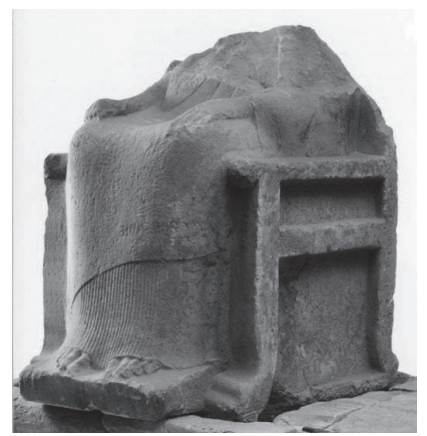

Fig. 28. Groupe de Généléos, Phileia,

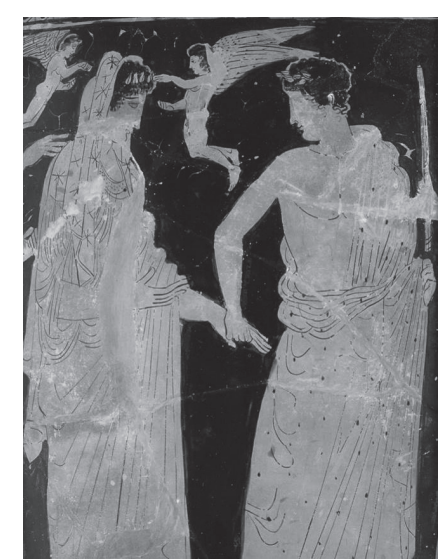

Fig. 30. Loutrophore attique à figures rouges, Musée des Beaux-Arts de Boston, inv. $n^{\circ}$ 03802, vers 430 av. J.-C. 
gravité en semble encore accusée. Notons en tout cas que la femme y apparaît d'une taille plus réduite, surtout sur le groupe du Louvre, où le contraste entre le visage de l'homme et celui de sa compagne s'accuse nettement. L'examen de tels objets rend plus sensible encore cette égalité déclarée et sereine, cette harmonie naturelle, bien vécue, que paraît renvoyer la contemplation des époux étrusques.

Pourquoi l'iconographie grecque ne reflète-t-elle pas de semblables images? Je ne vais certes pas m'engager dans un discours de civilisations comparées où l'histoire, la religion, la sociologie sont profondément concernées. Mais regardons seulement quelques représentations qui donnent à penser, et d'abord ces deux scènes de banquet, où la place et le rôle de la femme sont clairement illustrées : richement vêtue, digne et respectée sur la paroi de la “ Tombe des Léopards », la femme couchée sur la klinè, comme les hommes, y participe aux réjouissances $(\text { fig. 23 })^{20}$, tandis que sur le décor de quelques coupes attiques à figures rouges, elle est bien aussi couchée, mais cette fois nue et livrée au plaisir des hommes $(\text { fig. 24 })^{21}$. Certaines statuettes de femmes dans l'attitude du banquet couché, comme cette figurine attique de Berlin, confirment le trait par la nudité totale qui s'y affiche (fig. 25) ${ }^{22}$. Et deux autres groupes d'argile, l'un de Béotie (fig. 26), l'autre de l'Attique (fig. 27), avec quelques variations, s'inscrivent dans la même tradition ${ }^{23}$.

Oui, chez les Grecs la posture du banqueteur couché, au temps des sarcophages Campana, ne peut convenir à une femme honnête : elle est l'apanage de l'homme. Retournons donc à Samos, où un célèbre monument sculpté avant le milieu du $\mathrm{VI}^{\mathrm{e}}$ siècle av. J.-C. distribue leurs postures respectives très clairement : c'est le groupe dit de Généléos, du nom du dédicant, où, de part et d'autre d'une progéniture dont ils sont fiers, apparaissent d'un côté Phileia, la mère (fig. 28), trônant avec gravité, et de l'autre côté son époux, dont le nom n'est conservé qu'à moitié, et qui s'abandonne, à demi couché sur sa klinè, au repos et au plaisir du banquet (fig. 29 $)^{24}$. L'homme allongé sur la couche du banquet, la femme assise dans sa

20 R. Bianchi Bandinelli - A. Giuliano, les Etrusques et l'Italie avant Rome, Paris 1973, p. 266, fig. 304 .

21 J. Boardman, Athenian Red Figure Vases - The Classical Period, Londres 1989, p. 38 et p. 47, fig 72. P. Blome, Musée des Antiquités Classiques de Bâle et Collection Ludwig, Bâle 1999, p. 80-81, fig. 108.

22 R. Kekule von Stradonitz, éd., Die Antiken Terrakotten, III.1, Die Typen der figürlichen Terrakotten, 1, Berlin 1903, p. 191, fig. 4 (F. WinTER)

23 Groupe attique (?) en terre-cuite : jeune homme et hétaïre sur la klinè du symposion : F. W. Hamdorf, Hauch des Prometheus-Meisterwerke in Ton, Munich 1996, p. 82 fig. 104, et p. 90. Groupe béotien : Musée de Dresde, inv. Z.V. 720 a : Die Antiken Terrakotten, op.cit. (ici note 22), p. 192, fig.1.

24 Samos - The Archaeological Museums, op.cit. (ici note 2), p. 84-87. 
proximité, voilà une mise en scène qu'on retrouve constamment sur les reliefs votifs et funéraires. A propos de cette différence de traitement, on peut ici rappeler le passage bien connu du Contre Néaira du Pseudo-Démosthène, où le plaideur répartit bien les fonctions, avec ce qui peut nous apparaître aujourd'hui comme une muflerie d'un cynisme tranquille ${ }^{25}$ :

“Qu'est-ce que vivre en mariage avec une femme? C'est avoir d'elle des enfants, présenter les fils à la phratrie et au dème, donner les filles en mariage en qualité de père. Nous prenons une courtisane pour le plaisir, une concubine pour recevoir d'elle les soins journaliers qu'exige notre santé, nous prenons une épouse pour avoir des enfants légitimes et une fidèle gardienne de tout ce que contient notre maison. »

Ce sont certes des propos d'avocat, qu'il faut remettre dans leur contexte. Mais ils reflètent néanmoins un certain comportement social, qui n’est pas celui des Etrusques.

Assurément, les images grecques qui nous renseignent sur le mariage expriment clairement la domination de l'homme, en tant que xú oıos de celle qui devient son

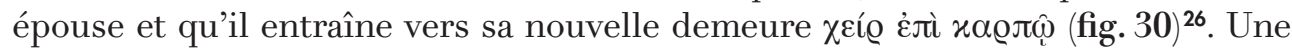

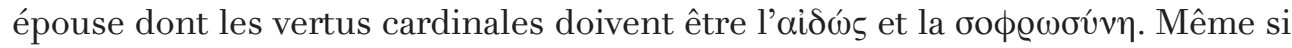
l'on doit éliminer définitivement l'idée d'un gynécée, où la femme grecque restait claustrée ${ }^{27}$, il n'en demeure pas moins qu'elle ne pouvait participer aux réunions des hommes, ni sortir librement. En résumant beaucoup sa situation, répétons qu'une femme, à Athènes, est l'épouse d'un Athénien, plutôt qu'une Athénienne. L'imagerie de la céramique montre bien, sur une pyxis attique à figures rouges (fig. 31), ce qui doit rythmer le cours de la vie des femmes : le lit qui apparaît par la porte entrouverte de la chambre, le miroir, “ conseiller de leur grâce », le métier à tisser, qui s'attache à leur « genre » (fig. 32), au point que les courtisanes doivent ajouter la compétence d'une bonne fileuse à d'autres talents plus spécifiques (fig. 33 ${ }^{\mathbf{2 8}}$. L’un des grands modèles féminins, pour les Grecs, c'est Pénélope, laquelle, toute réservée dans la tristesse que lui inspire l'absence de son époux, use de cet art de tisser pour n'avoir pas à le trahir (fig. 34).

25 Pseudo-Démosthène, Contre Néaira 122.

26 Voir, par exemple, outre la pyxis attique à figures rouges, Louvre L 55 attribuée au Peintre du Mariage ( $c f$. F. Lissarrague, Vases grecs, Paris 1999, p. 124, 125, 127, fig. 97-98) la loutrophore attique à figures rouges conservée à Boston, Museum of Fine Arts, inv. 03802 (cf. J. OAKLey -R. Sinos, The Wedding in Ancient Athens, Madison 1993, p. 105107, fig. 1).

27 Voir sur ce point les sages remarques de M.- Chr. Hellmann, L'architecture grecque, III, Paris 2010, p. 53-55.

28 Pyxide attique à figures rouges, Louvre CA 587 : cf. La cité des images, Mont-surLausanne 1984, p. 97, fig. 140 (Cl. BERARD). 


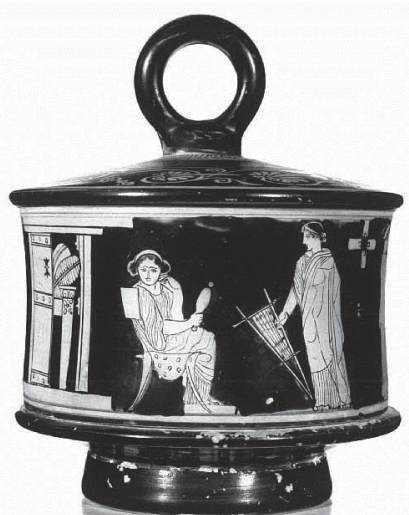

Fig. 31. Pyxis attique à figures rouges, Louvre CA 587, vers 430 av. J.-C.

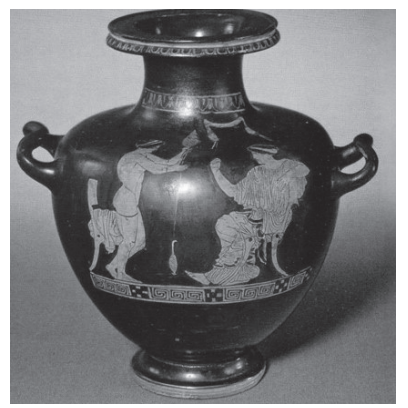

Fig. 33. Hydrie attique à figures rouges, Musée National de Copenhague, inv. $n^{\circ} 153$, vers 440 av. J.-C.
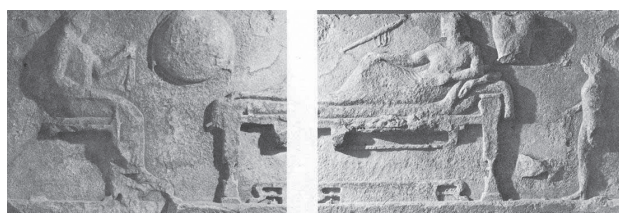

Fig. 35. Relief au banquet funèbre, Paros (Katapoliani) Musée de Paros, vers 500 av. J.-C.

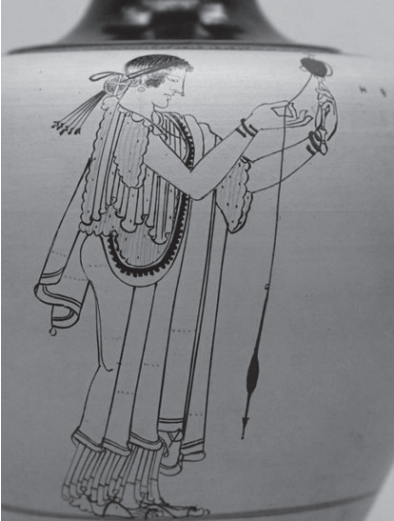

Fig. 32. Oenochoé attique à fond blanc, British Museum GR 1873.8-20.304, vers 490 av. J.-C.

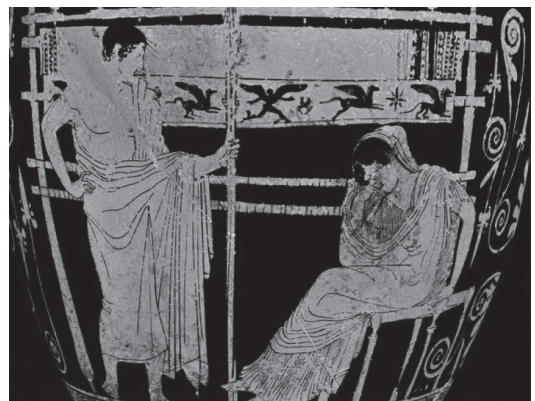

Fig. 34. Skyphos attique à figures rouges, Musée de Chiusi inv. 1831, vers 440 av. J.-C.

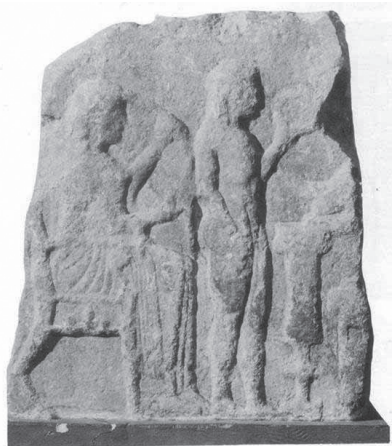

Fig. 36. Relief au banquet funèbre, Tégée, Musée National d'Athènes inv. $n^{\circ} 55$, vers 520 av. J.-C. 


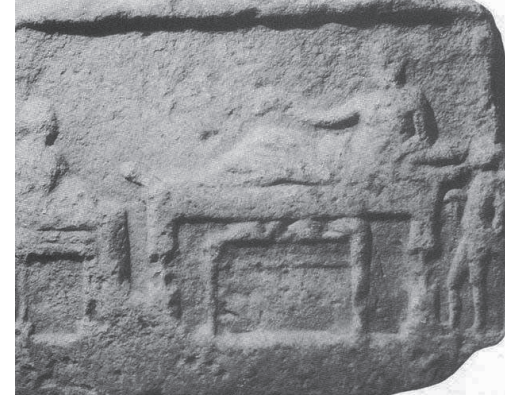

Fig. 37. Relief au banquet funèbre, Athènes, Musée National d'Athènes inv. $\mathrm{n}^{\circ}$ 4802, vers 450 av. J.-C. (?)

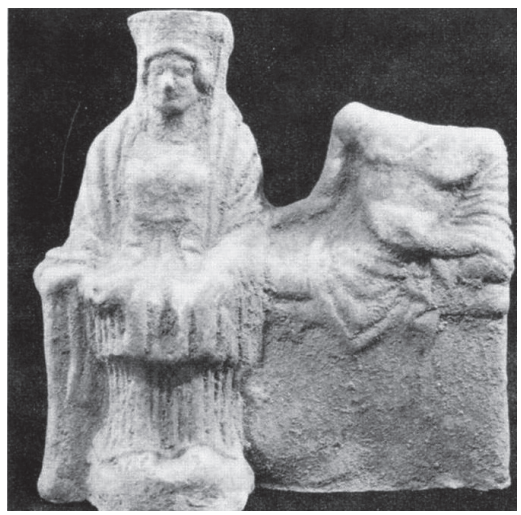

Fig. 39. Groupe en terre cuite de Tarente, Musée National de Tarente, inv. n²0011, vers le milieu du Ve siècle av. J.-C.

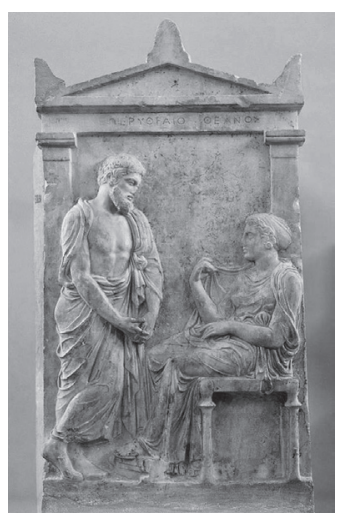

Fig. 41. Stèle de Ktesilaos et Theano, Musée National d'Athènes, inv. n 3472, vers 400 av. J-C.

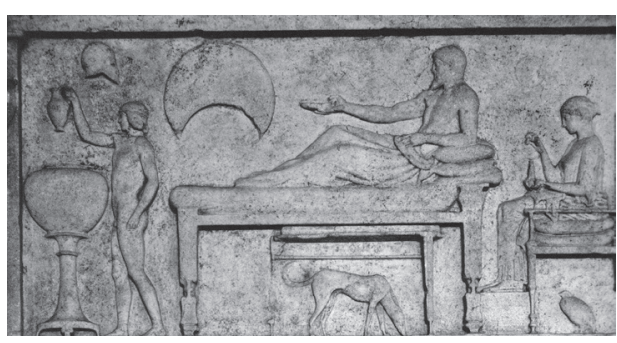

Fig. 38. Relief au banquet funèbre, Thasos, Musée Archéologique d'Istanbul.

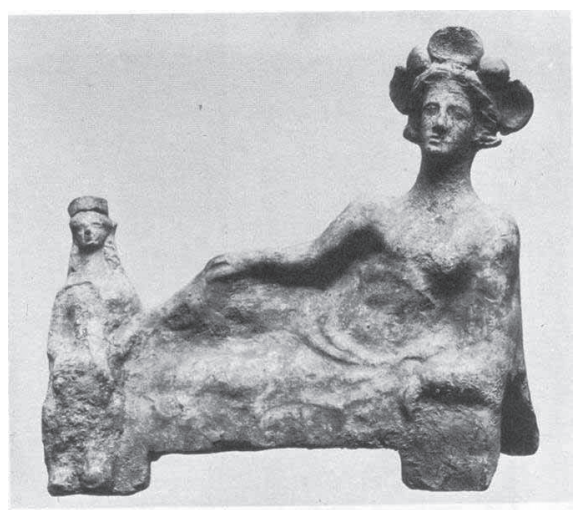

Fig. 40. Groupe en terre cuite de Tarente, Ashmolean Museum d'Oxford, n 1886.746, vers le milieu du IV siècle av. J.-C.

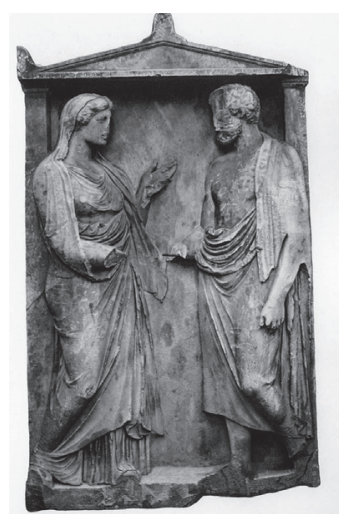

Fig. 42. Stèle de Mnèsistratè, Musée National d'Athènes, inv. n 826, vers 350 av. J.-C. 
Donc, l'homme allongé sur la couche du banquet, la femme assise dans sa proximité, voilà une mise en scène qu'on retrouve constamment sur les reliefs grecs votifs et funéraires, dont l'interprétation est souvent discutée ${ }^{29}$. Sans entrer dans ce débat, jetons les yeux sur quelques-uns d'entre eux, et d'abord ceux qui ne s'éloignent pas trop de la date des sarcophages de ce colloque, du dernier tiers du $\mathrm{VI}^{\mathrm{e}}$ au début du $\mathrm{V}^{\mathrm{e}}$ siècle : un relief de Tégée (fig. 36), un autre d'Athènes (fig. 37), un troisième de Paros (fig. 35) ${ }^{30}$. De 530 à 480 av. J.-C., ils regroupent, en y ajoutant l'échanson, les trois éléments de la composition : l'homme, le lit du banquet et la femme, qui exécute au moins deux fois le geste du dévoilement. Mais par trois fois, elle reste assise au pied du lit. Un célèbre relief de Thasos, au musée d'Istanbul, garde les acteurs, en changeant la place de la femme, qui, toujours assise, est passée derrière le lit, où elle manipule un vase à parfum, comme les épouses en terre cuite de Cerveteri (fig. 38$)^{31}$.

L'homme au banquet, avec une femme assise sur le pied du lit, c'est là un schéma incessamment répété par une grande classe d'objets en terre cuite, qui se rencontrent de la fin du VI jusqu'à la fin du IV siècle, celle des reliefs au banquet trouvés en masse à Tarente (fig. 39), qui ne sont pas sans rapport avec les reliefs de Sparte, dont Tarente est la colonie ${ }^{32}$. Là encore l'interprétation varie d'un savant à un autre : banquet divin, banquet de héros mythologiques, banquet de mortels héroïsés. Voyons-y seulement, aujourd'hui, l'image d'un homme banquetant, souvent coiffé d'un couvre-chef richement orné, tandis que la femme qui l'accompagne est sagement assise au pied du lit, parfois réduite à un motif diminutif (fig. 40). Nous sommes bien loin du spectacle offert par les époux des sarcophages de Caeré.

Les réflexions que nous avons menées sur l'iconographie du couple dans l'art grec devraient être poursuivies dans le domaine des stèles funéraires, surtout à Athènes, stèles qui ne manquent pas de reliefs où le mari et la femme sont représentés ${ }^{33}$. On se bornera ici à quelques phrases et à quelques images, car,

29 Voir J.- M. Dentzer, Le motif du banquet couché dans le Proche-Orient et le monde grec du VII ${ }^{e}$ au IV siècle av. J.-C., Paris - Rome 1982, p. 298-299 et p. 559-566.

30 Tégée : Dentzer, ibid., p. 252-262.

31 Br. Sismondo - Ridgway, " The Banquet Relief from Thasos », American Journal of Archaeology 71, 1967, p. 307-309.

32 Cf. H. Herdejürgen, Die Tarentinischen Terrakotten des 6. bis 4. Jahrhunderts v. Chr Katalog der Terrakotten im Basler Antikenmuseum, Bâle 1971 ; A. Bencze, Physionomie d'une cité archaïque : développement stylistique de la coroplathie votive archaïque de Tarente, Naples - Centre J. Bérard, 2013.

33 Sur les stèles funéraires archaïques, G. M. A. Richter, The Archaic Gravestones of Attica, Londres 1961 ; sur les stèles classiques, voir la synthèse de Chr. C. Cuatrmont, 
d'une part, la structure de ces monuments funéraires s'éloigne de la formule du sarcophage, et d'autre part ces couples sculptés appartiennent pour la plupart à l'époque classique. Qu'y voit-on? Le plus souvent un homme debout et une femme assise (fig. 41) ou debout (fig. 42), avec des noms qui ne permettent que rarement de savoir avec certitude qui est mort, et surtout où le rapport époux-épouse n'est pas toujours assuré. Sur ces images inévitablement formulaires, des épitaphes sont parfois gravées, sans que la relation entre l'inscription et les personnages représentés soit évidente. Et la sculpture de ces stèles pouvait d'ailleurs anticiper l'usage qu'on en faisait, et donc n'avoir qu'un rapport très lâche avec la réalité d'un décès précis. Toutes ces réserves mises à part, peut-on y retrouver les signes d'un attachement conjugal, ou en tout cas d'une harmonie comparable à celle que les sarcophages étrusques semblent donner à voir? Certes non. La répétition régulière des mêmes motifs, tel celui de la dexiosis, le serrement de mains, en réduit l'effet à celui d'une convention mécanique, même si ce serrement de mains, dont le premier exemple remonte jusqu'à la fin de l'archaïsme (fig. 43) ${ }^{34}$, peut parfois être enrichi de détails inhabituels, comme au Louvre,où l'homme se sert de ses deux mains (fig. 44). Notons par ailleurs l'existence d'une série de représentations qui utilisent la scène du banquet funèbre : on y retrouve alors l'homme à demi couché sur sa klinè, et la femme assise au bout du lit (fig. 45 $)^{35}$.

Telles sont donc les réflexions, bien rapidement et bien légèrement esquissées, que peuvent inspirer les représentations du couple homme-femme au sein de l'art grec. Mais je ne voudrais pas clore cette intervention sans mentionner d'une part l'exemple particulier des deux statues déliennes de Dioscourides et Cléopâtra $(\text { fig. 46 })^{36}$, groupe commémoratif que cette dernière avait fait exécuter juste après 138 av. J.-C., pour rappeler d'une manière bien officielle, peut-être même un peu trop, la piété de son mari ; et d'autre part le groupe en terre cuite trouvé dans les fouilles de Myrina (fig. 47), où l'on retrouve la klinè, avec cette fois deux jeunes gens ${ }^{37}$. La couche est luxueuse, et l'empressement du jeune homme d'une tendresse impatiente. C'est le moment de l'anakalypsis, le dévoilement de celle qui va devenir épouse, dans la chambre nuptiale.

Classical Attic Tombstone (7 volumes plus un supplément), Kilchberg 1993. Les reliefs où seraient représentés les couples mari et femme se trouvent groupés dans le volume II, p. $45-48$.

34 Stèle funéraire du musée d'Egine, où ce sont deux mains de femmes qui sont jointes : cf. E. Berger, Das Baslerarzt Relief, Bâle 1970, p. 110-111, fig. 132. Sur la réalité de la tendresse conjugale, voir G. Raepset, "Sentiments conjugaux à Athènes aux V $\mathrm{V}^{\mathrm{e}}$ et $\mathrm{IV}^{\mathrm{e}}$ siècles avant notre ère ", l’Antiquité Classique 150, 1981, p. 677-684. Voir Dentzer, op. cit. (ici note 29), pl. 78, fig. 466-469.

36 Delos, op.cit. (ici note 14), p. 161 et p. 276.

37 S. Besques, Figurines et reliefs grecs en terre cuite, Paris 1994, p. 33 et p. 96-97, fig. 90. 


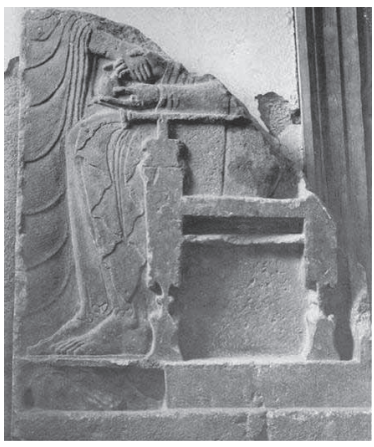

Fig. 43. Fragment de stèle avec dexiosis, Musée d'Egine, vers 490 av. J.-C.

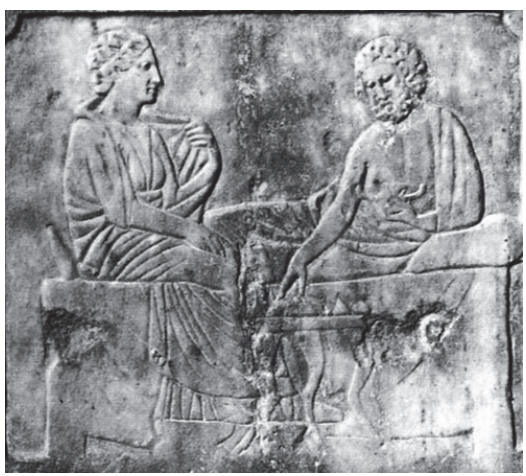

Fig. 45. Stèle de Pyrrhias et Thettalè, Musée

National d'Athènes inv. n 897 , vers 340 av. J.-C.

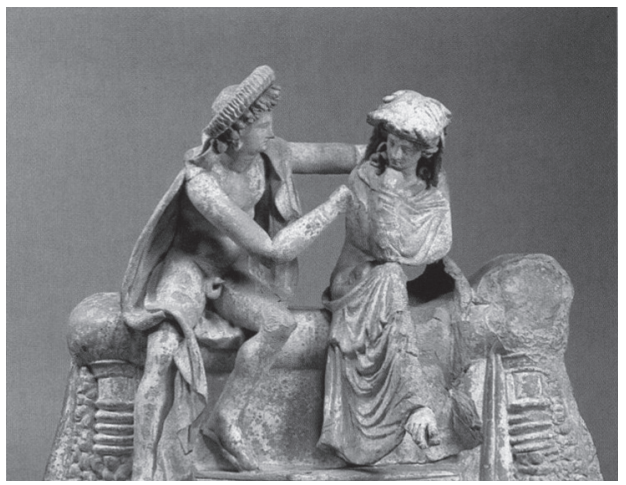

Fig. 47. Couple sur le lit nuptial, Myrina, Louvre MYR 268, vers 150-100 av. J.-C.

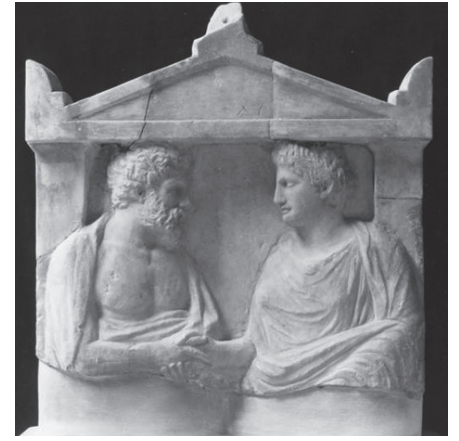

Fig. 44. Fragment de stèle avec dexiosis, Louvre LL 66, vers 400 av. J.-C. (et détail)

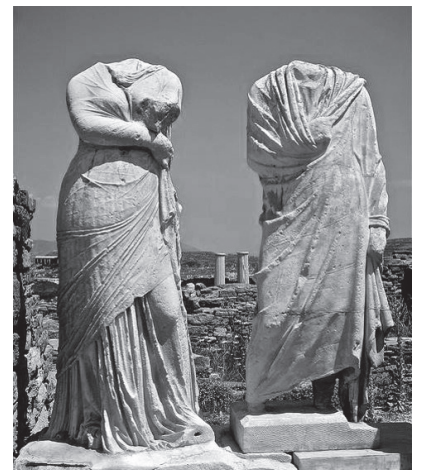

Fig. 46. Statues de Cléopâtre et

Dioskouridès, Musée de Délos A 7763, 7799, 7997a, vers 138/137 av. J.-C.

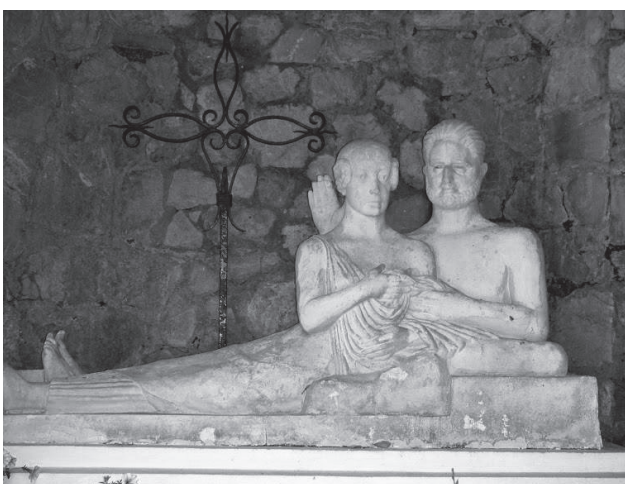

Fig. 48. Sarcophage de Jean-Gabriel

Domergue et de son épouse Odette Maudrange, sculpté par celle-ci après 1932 pour leur villa de Cannes 
Entre la gravité solennelle des deux statues exposées dans la maison de Délos et l'atmosphère troublante qui nimbe le groupe gracieux de Myrina, on pourrait presque dire que les époux des sarcophages Campana trouvent leur place : dignes sans être guindés, tendrement unis, sans trop d'abandon.

Et je voudrais, pour terminer, montrer l'image d'un groupe sculpté dont la relation avec le sarcophage du Louvre est d'une évidente clarté. Ce groupe, ce n'est pas dans l'Antiquité grecque que je l'ai rencontré, mais dans la villa d'un peintre, Jean-Gabriel Domergue, construite à Cannes dans les années $30 \mathrm{du} \mathrm{XX}^{\mathrm{e}}$ siècle : son épouse, Odette Maudrange, artiste sculpteur, avait créé pour le couple qu'ils formaient un sarcophage en pierre, placé dans leur jardin, qui s'inspirait directement du tombeau étrusque (fig. 48) ${ }^{38}$. Leurs effigies sont bien plus raides et plus sévères qu'à Cerveteri. Peut-être Madame Domergue avait-elle voulu marquer ainsi une certaine distance avec l'esprit des peintures de son époux, où les petites femmes de Paris se montrent plus que coquettes...

\author{
Alain Pasquier \\ Conservateur général honoraire \\ du patrimoine \\ 121 boulevard Jean-Jaurès \\ 92100 Boulogne \\ pasquieraj@yahoo.fr
}

38 Cannes, Villa Fiesole, devenue Villa Domergue : voir, par exemple, le site Internet www.theprovenceherald.fr, Culture, Art/Expositions. 